\title{
Influence of the Metal Substrate on the Adsorption Properties of Thin Oxide Layers: Au Atoms on a Thin Alumina Film on NiAl(110)
}

\author{
M. Kulawik, N. Nilius, ${ }^{*}$ and H.-J. Freund \\ Fritz-Haber-Institut der Max-Planck-Gesellschaft, Faradayweg 4-6, D-14195 Berlin, Germany
}

(Received 19 July 2005; published 24 January 2006)

\begin{abstract}
Thin oxide films grown on metal substrates are widely used in surface science to model bulk oxides, assuming their chemical and electronic properties to be similar. In some cases, however, this might not be justified as the present scanning tunneling microscopy studies demonstrate for Au atoms on a thin alumina film on $\mathrm{NiAl}(110)$. Au atoms were evaporated onto the oxide film at a sample temperature of $\sim 10 \mathrm{~K}$. At low coverage, this leads to the formation of one-dimensional clusters with unusually large $\mathrm{Au}-\mathrm{Au}$ distances of 5.6-6.0 $\mathrm{\AA}$. A direct interaction between the $\mathrm{Au}$ atoms can be excluded, and a substratemediated mechanism is supposed instead. This assumption is strengthened by the finding that the $\mathrm{Au}$ chains exhibit a preferential orientation: They are almost aligned with the [001] direction of the $\mathrm{NiAl}(110)$ substrate, clearly indicating that the metal substrate participates in the binding of the Au atoms.
\end{abstract}

DOI: 10.1103/PhysRevLett.96.036103

Metal particles on thin oxide films are well established in surface science to model heterogeneous catalysts [1-3]. These model systems have similar chemical and electronic properties as real catalysts but are by far less complex and easier to investigate because of the conductivity of thin films in contrast to most bulk oxides. Oxide-film-based model systems are, therefore, well-suited to gain fundamental understanding on the nature of catalytically active sites and reaction kinetics [4]. Other questions concern the material transport on oxide surfaces and aim to determine preferential adsorption sites, diffusion, nucleation, and catalytic activity of supported metal atoms.

Commonly, thin oxide films with band gaps similar to the corresponding bulk oxides (usually wide-gap insulators) are supposed to reflect also their chemical properties and to be, therefore, suited as model systems for the bulk material. This is justified by a huge amount of experimental evidence [1-4]. However, according to a recent theoretical work, a generalization of this assumption to all metal-oxide-film systems has to be doubted [5]. The authors compared the adsorption of $\mathrm{Au}$ atoms on bulk $\mathrm{MgO}$ with that on $\mathrm{MgO}$ films on $\mathrm{Mo}(100)$. While Au atoms were found to be neutral on bulk $\mathrm{MgO}$, they appeared to be negatively charged on thin $\mathrm{MgO}$ films. The effect was ascribed to electron tunneling from the underlying metal substrate $[5,6]$, induced by the high electron affinity of $\mathrm{Au}$ $(2.31 \mathrm{eV})$. This theoretical study suggests that careful analysis is necessary to evaluate the suitability of thin films as model support. However, to our knowledge, experimental evidence for such deviating properties of thin oxide films has not been reported yet. In this Letter, we provide scanning tunneling microscopy (STM) studies which clearly reveal an influence of the limited film thickness on the adsorption behavior of an oxide film. For that purpose, we investigated the nucleation of Au atoms on a $5 \AA$ thin alumina film on $\operatorname{NiAl}(110)[7,8]$ with STM. The film has a band gap of $\sim 8 \mathrm{eV}$, which is similar to that of bulk alumina $(8.8 \mathrm{eV})$ [8]. Its structure was resolved only
PACS numbers: 68.43.Hn, 68.37.Ef, 68.47.Jn, 73.22.-f

recently [9], revealing the stoichiometry to be $\mathrm{Al}_{10} \mathrm{O}_{13}$ and opening new possibilities for the understanding of the interaction with metal adatoms. Surprisingly, well-defined one-dimensional Au clusters are found upon deposition at $\sim 10 \mathrm{~K}$. Their orientation clearly indicates an involvement of the NiAl substrate in the binding of the Au atoms.

The measurements were performed in ultrahigh vacuum, using a home-built STM operated at $5 \mathrm{~K} \mathrm{[10].} \mathrm{The} \mathrm{clean}$ $\mathrm{NiAl}(110)$ surface is prepared by repeated cycles of $\mathrm{Ar}^{+}$ sputtering and annealing to $1000^{\circ} \mathrm{C}$. Subsequent dosing of $\mathrm{O}_{2}$ at $280^{\circ} \mathrm{C}$ and annealing to $800^{\circ} \mathrm{C}$ leads to the formation of a thin, crystalline alumina film [7]. Au is evaporated from a gold-covered tungsten wire onto the sample, which has then a temperature of $\sim 10 \mathrm{~K}$.

A typical coverage used for our studies is shown in the STM image in Fig. 1. The image reveals the presence of both $\mathrm{Au}$ monomers and clusters, which form even at low coverage because $\mathrm{Au}$ atoms can move on the surface prior to thermalization. While Au aggregates are imaged with a large apparent diameter at positive sample bias $V_{S}$, they appear much smaller at negative $V_{S}$, allowing the determination of their internal structure. The majority are $\mathrm{Au}$ dimers with different orientations, but also small clusters are observed. Surprisingly, many of these clusters are onedimensional (1D) chains. Figure 2 shows STM images of a $\mathrm{Au}$ monomer and such self-assembled $\mathrm{Au}$ chains with a maximum length of $22.5 \AA$. Longer chains have not been observed. The images are recorded at negative $V_{S}$, whereby $V_{S}$ is chosen well above (left side) and close to (middle) the highest occupied state of the Au chains, which was determined by scanning tunneling spectroscopy (STS). On the left-hand side, the chains appear with a uniform height of $\sim 4 \AA$. The imaging properties change dramatically in the case of resonant tunneling into the electronic states of the chain (middle). The apparent height of the chains is then increased by $1-2 \AA$. Furthermore, the chains show a distinct substructure, consisting of 3-5 lobes. These lobes are separated by $5.6-6.0 \AA$, which corresponds to the double 


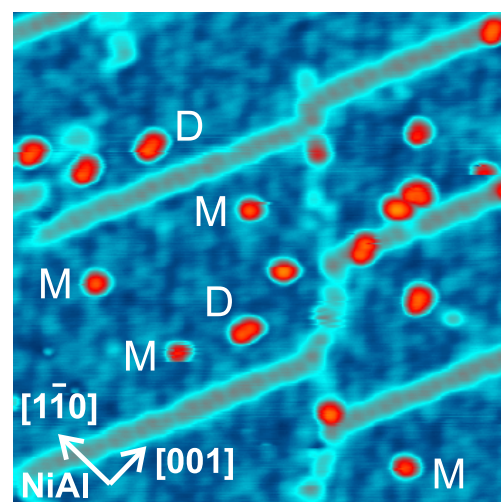

FIG. 1 (color online). STM image showing Au adatoms and clusters on a thin alumina film on $\operatorname{NiAl}(110)(430 \AA \times 430 \AA$, $\left.V_{S}=3.0 \mathrm{~V}\right)$. At the displayed coverage, mostly monomers (M) and dimers (D) are found. The bright stripes are domain boundaries, regularly appearing line defects of the oxide film $[12,15]$. The marked orientation of the $\mathrm{NiAl}$ substrate is the same in all STM images of this work.

$\mathrm{Al}-\mathrm{Al}$ distance in the surface aluminum $\left(\mathrm{Al}_{S}\right)$ layer of the thin oxide film [9]. We suggest that each lobe reflects the position of a $\mathrm{Au}$ atom or a Au-induced electronic state, because no intermediate chain lengths have been observed. Thus, the chains in Fig. 2 would correspond to dimers, trimers, tetramers, and pentamers. This interpretation implies an untypical Au-Au distance, which would be about twice as large as in bulk gold $(2.9 \AA)$ or in gas phase dimers (2.5 А) [11].

The Au chains on the alumina film show a preferential orientation which is close to the $\mathrm{NiAl}$ [001] direction. This fact is especially surprising, since the oxide film grows in two domains $A$ and $B$, which are rotated against each other by $48^{\circ}$ [Figs. 3(c) and 3(d)]. Despite this very different orientation of the alumina substrate, the direction of the $\mathrm{Au}$ chains differs only little in both domains, as shown for two pentamers in Figs. 3(a) and 3(b). The angle between the chain axis and the $\mathrm{NiAl}$ [001] direction is characteristic in each domain: It amounts to $+5^{\circ}$ in the $A$ domain and $-5^{\circ}$ in the $B$ domain. This observation can be rationalized as follows. The $\mathrm{Al}_{S}$ layer of the oxide film forms a quasihexagonal lattice [9], whereby its orientation differs in domains $A$ and $B$ by an angle of $60^{\circ}-48^{\circ}=12^{\circ}$. This is visualized in Figs. 3(c) and 3(d) for a perfectly hexagonal and, thus, simplified $\mathrm{Al}_{S}$ lattice (gray circles). The $\mathrm{Au}$ chains form along that vector of the $\mathrm{Al}_{S}$ lattice which is closest to the $\mathrm{NiAl}$ [001] direction. The maximum angle between both is $\pm 6^{\circ}$, dependent on the domain, which agrees well with the experimentally determined value of $\pm 5^{\circ}$. The slight difference can be explained with the deviation of the $\mathrm{Al}_{S}$ lattice from the perfectly hexagonal arrangement (cf. Ref. [9]). The misalignment between the chain axis and the NiAl [001] direction also provides a plausible explanation for the limited chain length of $22.5 \AA$ : Apparently, a favorable constellation between the $\mathrm{Al}_{S}$ lattice and the close-packed rows of NiAl exists only
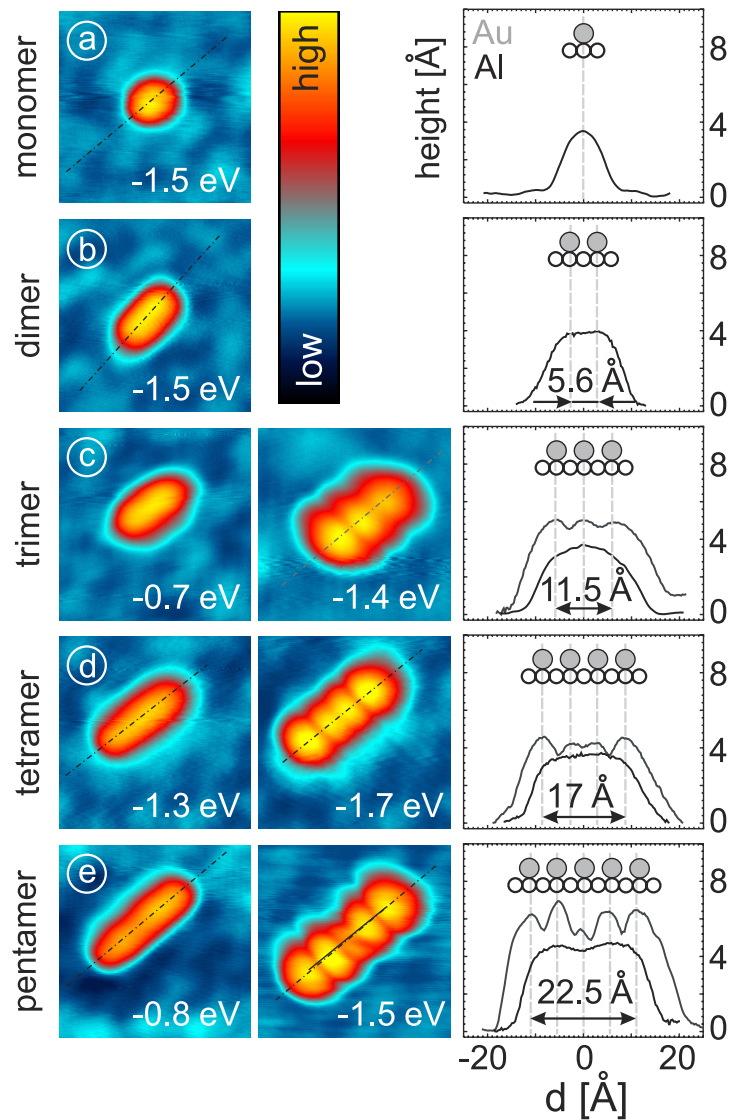

FIG. 2 (color online). STM images (43 $\AA$ ×43 $\AA$ ) and according height profiles of a $\mathrm{Au}$ monomer (a) and self-assembled $\mathrm{Au}$ chains containing two to five atoms [(b)-(e)] on an alumina film on $\mathrm{NiAl}(110)$. The STM images are recorded at negative sample bias $V_{S}$, whereby $V_{S}$ is chosen either well above (left side) or close to (middle) the highest occupied state of the Au chain, as determined by STS.

for some adsorption sites. Already $12 \AA$ away from the optimum position, the divergence is $\sim 1 \AA$, hampering the elongation of the chain. This is shown schematically in Figs. 3(c) and 3(d).

Figures 3(e) and 3(f) give further insight into the arrangement of the $\mathrm{Au}$ chains with respect to the oxide surface. Both STM images show the same Au trimer, once imaged with a metallic tip [3(f)] and once with a tip modified by an adsorbate, leading to supercorrugation and atomic resolution [3(e)]. The atomically resolved lattice corresponds to the $\mathrm{Al}_{S}$ lattice, as deduced from its quasihexagonal symmetry and the number of atoms (24) per unit cell $[9,12]$. The image clearly confirms the alignment of the Au chain and $\mathrm{Al}_{S}$ rows. Furthermore, we can extrapolate the atom positions of the $\mathrm{Al}_{S}$ lattice to the area underneath the $\mathrm{Au}$ trimer to identify the $\mathrm{Au}$ adsorption sites. The middle atom of the trimer is located on top of an $\mathrm{Al}_{S}$ atom, while the end atoms appear to be adsorbed on $\mathrm{Al}$ bridge sites. However, the positions of the end atoms might be altered by a slip-stick motion of the adsorbate on the tip when scanning over the trimer, because $\mathrm{Au}-\mathrm{Au}$ distances 

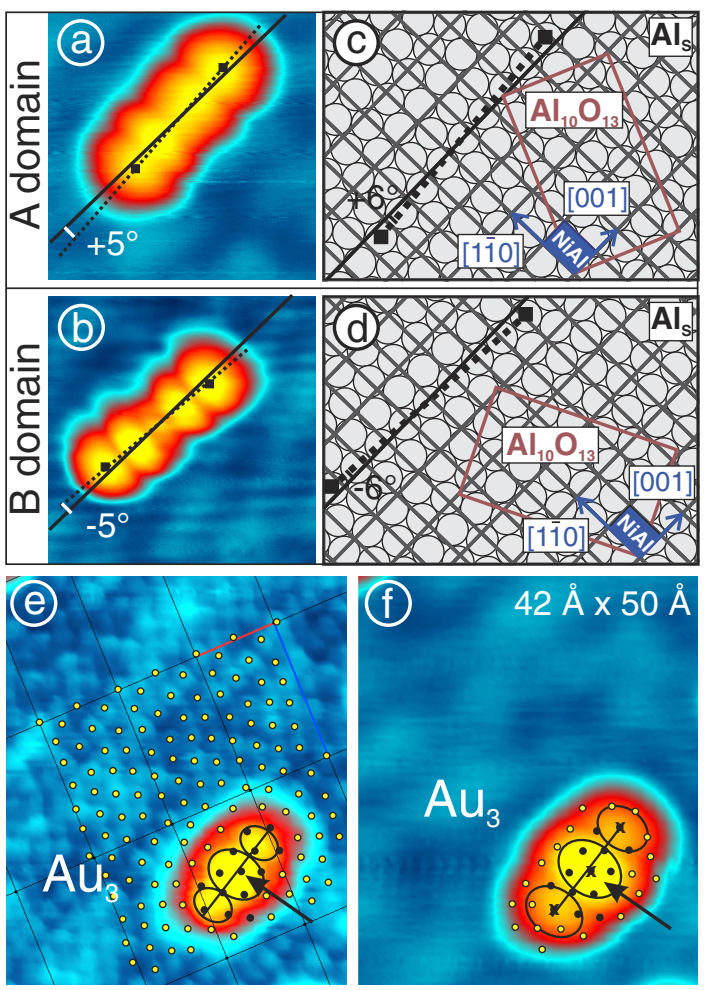

FIG. 3 (color online). (a)-(b) STM images $(47 \AA \times 47 \AA$, $V_{S}=-1.8 \mathrm{~V}$ ) of $\mathrm{Au}$ pentamers on the alumina film on $\mathrm{NiAl}(110)$, which are adsorbed in the $A$ and $B$ domains, respectively. The angles between the chain axis (dotted lines) and the $\mathrm{NiAl}$ [001] direction (straight lines) are indicated. (c)(d) Schemes showing the orientation of the (idealized) hexagonal $\mathrm{Al}_{S}$ lattice (gray circles) with respect to the $\mathrm{NiAl}$ substrate. The dotted lines reflect the extension and orientation of Au pentamers. The unit cell parameters are $\left(a_{1}=4.08 \AA, a_{2}=2.89 \AA\right)$ for $\mathrm{NiAl}$ and $\left(b_{1}=17.88 \AA, b_{2}=10.55 \AA, \alpha=89^{\circ}\right)$ for the alumina film. (e) STM image showing a Au trimer $\left(V_{S}=-0.7 \mathrm{~V}\right)$ and the $\mathrm{Al}_{S}$ layer with atomic resolution due to supercorrugation. The atom positions are marked, whereby the lattice is extrapolated to the area underneath the Au trimer. (f) STM image of the same region with a metallic tip at $V_{S}=-1.2 \mathrm{~V}$. The lattice from (e) is superposed.

are considerably shorter than in normal images [cf. Figs. 2 and 3(f)]. If the lattice is superposed onto the STM image without supercorrugation [Fig. 3(f)], the adsorption site would be the same for all atoms of the trimer, i.e., on top of $\mathrm{Al}_{S}$. Calculations for $\mathrm{Au}$ atoms on $\alpha-\mathrm{Al}_{2} \mathrm{O}_{3}$ predict almost equal adsorption energies for $\mathrm{O}$ and $\mathrm{Al}$ on-top sites with a small preference for $\mathrm{O}(0.03 \mathrm{eV})$ [13]. However, the situation can change dramatically for thin oxide films, as demonstrated recently for $\mathrm{Au}$ on $\mathrm{MgO}$. While adsorption occurs preferentially on top of $\mathrm{O}$ for bulk $\mathrm{MgO}$, cation sites are favorable on thin $\mathrm{MgO}$ films on $\mathrm{Mo}(100)$ [5].

The results described thus far indicate unambiguously the participation of the $\mathrm{NiAl}$ substrate in the binding of $\mathrm{Au}$ adatoms: Au chains form along the close-packed rows of $\mathrm{NiAl}$, whereby the mean $\mathrm{Au}-\mathrm{Au}$ distance is compatible with twice the $\mathrm{Al}_{S}-\mathrm{Al}_{S}$ distance and twice the $\mathrm{Ni}-\mathrm{Ni} /$
Al-Al separation in the metal support. Apparently, the most stable $\mathrm{Au}$ adsorption sites on the alumina film are the ones where $\mathrm{Al}_{S}$ atoms and atoms in $\mathrm{Ni}$ or $\mathrm{Al}$ rows have a distinct arrangement, for instance, on top of each other. Such preferential adsorption sites are relatively rare and appear randomly on the surface because of the incommensurate growth and the rotation of the alumina lattice with respect to $\mathrm{NiAl}(110)[7,8]$.

The lobe structure of the Au chains suggests an interatomic distance of 5.6-6.0 $\mathrm{A}$, which is roughly twice the $\mathrm{Au}-\mathrm{Au}$ distance in bulk gold. Certainly, the questions arise whether the observed lobes do indeed correspond to $\mathrm{Au}$ adatoms and why Au atoms would not be located on adjacent oxide adsorption sites, particularly because they would be separated by $\sim 3.0 \AA$. To address these points, we have performed STS and conductance $(d I / d V)$ imaging. Figure 4 summarizes the STS data of a Au pentamer in the range between -2.2 and $3.4 \mathrm{~V}$. Higher negative or positive $V_{S}$ usually led to the desorption of the pentamer or parts of it. At negative $V_{S}$, two resonances can be detected by STS. They are located at each of the five lobes [Fig. 4(a), spectrum I] and almost vanish in between the lobes (spectrum II). This finding is confirmed by the $d I / d V$ images in Fig. 4(b), revealing the same symmetry for both negative resonances [14]. At positive $V_{S}$, three states at 1.8, 2.5, and $3.3 \mathrm{~V}$ [14] can be identified that are localized only at specific parts of the chain [Figs. 4(c) and 4(d)]. The spectroscopic data observed for different Au pentamers on the alumina film are very similar with regard to energy and localization of the discrete states. They could be obtained repeatedly with different tips, for both oxide domains and different sample preparations. An influence of the tip can, therefore, be excluded. Furthermore, this finding indicates a narrow variation of adsorption sites for Au pentamers.

The spectroscopic data cannot be explained with the model of a dense-packed Au chain, sitting relatively decoupled on the alumina film. Such dense-packed Au and $\mathrm{Cu}$ chains (with interatomic distances close to the bulk metal) were found to show particle-in-a-box-like behavior on metal surfaces; i.e., a standing-wave pattern appeared in $d I / d V$ images exhibiting an increasing number of maxima and minima with increasing $V_{S}$ [16-18]. The $d I / d V$ patterns observed here are independent of $V_{S}$ for filled states and rather asymmetric for empty states. If the electronic properties were dominated by direct orbital overlap of $\mathrm{Au}$ adatoms on neighboring lattice sites, a stronger dispersion of electronic states and a clear standing-wave pattern would be expected. We therefore assign the electronic structure observed along $\mathrm{Au}$ chains to the signature of adatom-substrate interactions, only weakly modified by interadsorbate coupling. The chain formation would then be a result of the linear arrangement of favorable adsorption sites on the film, and the large Au adatom separation would not be in contradiction to typical Au-Au distances. Following this idea, the localized states below the Fermi level might be interpreted as defect states induced in the 

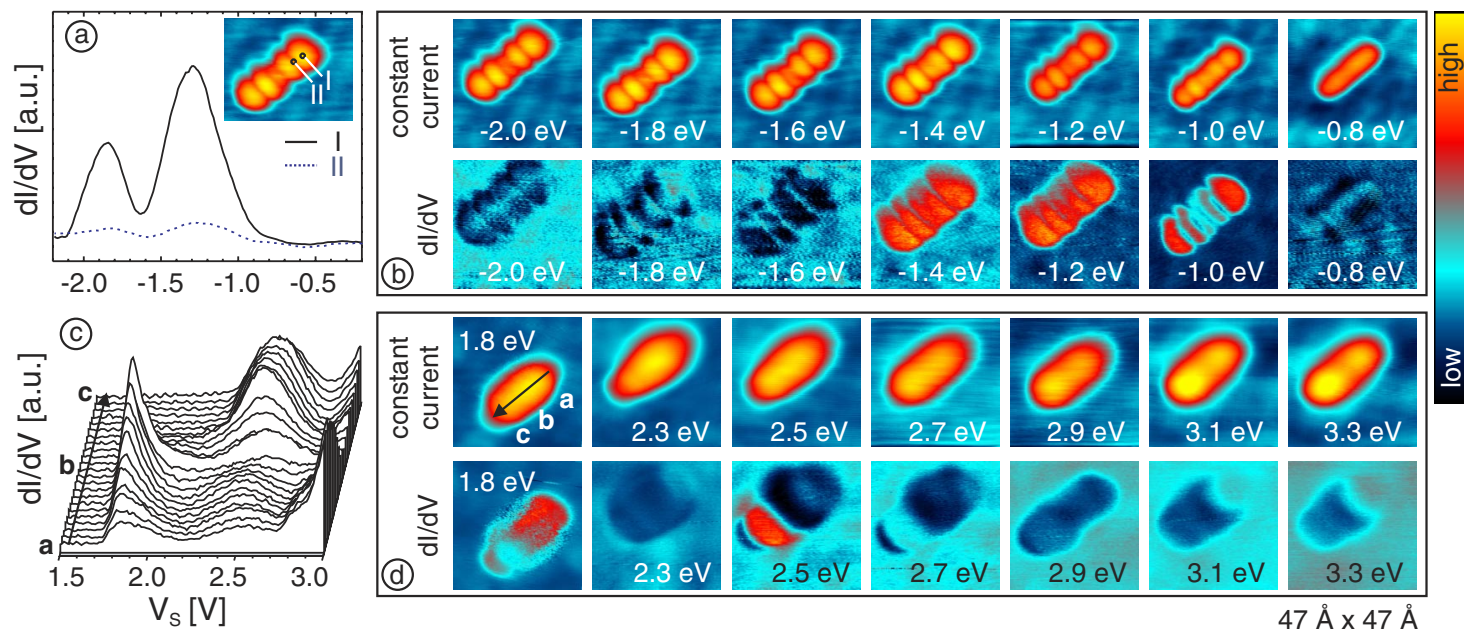

FIG. 4 (color online). Conductance spectra, constant current, and conductance $(d I / d V)$ images of a Au pentamer on the alumina film on $\mathrm{NiAl}(110)$. For better visualization, the intensity of the $d I / d V$ images was scaled. (a) At negative sample bias $V_{S}$, two states are detected, which are localized at each of the five lobes visible in the inset (position I). In between the lobes, nodal planes with strongly reduced conductance are observed (position II). The symmetry of these states is also reflected in the $d I / d V$ images of (b) [14]. (c) Conductance spectra taken at positive $V_{S}$ differ along the axis of the Au chain. (d) According conductance images.

alumina band gap by Au adsorption. The binding process might be responsible for modifications in the oxide lattice such as geometric relaxations, reorganization of chemical bonds, and/or charge transfer to the Au. Similar adsorbateinduced effects have been predicted for single metal atoms on oxide surfaces $[5,19,20]$, and it is known that lattice perturbations can cause defect states in the gap of oxide films $[12,15,21]$. The unoccupied states would then be the remnant of $\mathrm{Au}$ adatom states weakly interacting with each other, whereby their asymmetry reflects different local environments of $\mathrm{Au}$ atoms on the substrate. The remaining question, why Au adsorption on adjacent sites is suppressed on the alumina film, might be related to the repulsive nature of the above mentioned lattice modifications. Au-induced lattice defects might repel each other via polaronic or Coulomb interactions, causing a small barrier for the occupation of adjacent adsorption sites. Only at higher temperatures and increased Au coverage, adatoms can overcome this barrier and $3 \mathrm{D}$ clusters form on the alumina film.

In summary, we have investigated the adsorption of $\mathrm{Au}$ atoms on a thin alumina film on $\operatorname{NiAl}(110)$. Surprisingly, self-assembled chains with a maximum length of $22.5 \AA$ and a preferential orientation close to the [001] direction of the underlying $\mathrm{NiAl}(110)$ have been observed. These properties as well as spectroscopic data clearly indicate a participation of the NiAl substrate in the Au binding. Though a profound mechanistic understanding still requires theoretical investigations, our results show that the picture of inert thin oxide films does not hold in all cases, notably not towards metal adsorbates with high electron affinity, as already suggested by a theoretical prediction [5]. Accordingly, thin oxide films are (though wide-gap insulators) not generally suited to model bulk phases, and a careful analysis is necessary for each model system.
We thank H.-P. Rust and G. Kresse for fruitful discussion. M.K. gratefully acknowledges support from the Studienstiftung des deutschen Volkes.

*Electronic address: nilius@fhi-berlin.mpg.de

[1] C. T. Campbell, Surf. Sci. Rep. 27, 1 (1997).

[2] M. Bäumer and H.-J. Freund, Prog. Surf. Sci. 61, 127 (1999).

[3] W. T. Wallace, B. K. Min, and D. W. Goodman, Top. Catal. 34, 17 (2005).

[4] J. Libuda and H.-J. Freund, Surf. Sci. Rep. 57, 157 (2005).

[5] G. Pacchioni, L. Giordano, and M. Baistrocchi, Phys. Rev. Lett. 94, 226104 (2005).

[6] N. F. Mott, Trans. Faraday Soc. 43, 429 (1947).

[7] R. M. Jaeger et al., Surf. Sci. 259, 235 (1991).

[8] J. Libuda et al., Surf. Sci. 318, 61 (1994).

[9] G. Kresse et al., Science 308, 1440 (2005).

[10] H.-P. Rust et al., Rev. Sci. Instrum. 68, 129 (1997).

[11] CRC Handbook of Chemistry and Physics, edited by R. C. Weast (CRC Press, Cleveland, OH, 1974), 55th ed.

[12] M. Kulawik et al., Phys. Rev. Lett. 91, 256101 (2003).

[13] N. C. Hernández and J. F. Sanz, Appl. Surf. Sci. 238, 228 (2004).

[14] At higher positive or negative sample bias, the conductance of the bare alumina film [15] becomes similar to the value of the Au chains, resulting in vanishing or reversed contrast in $d I / d V$ images.

[15] N. Nilius et al., Phys. Rev. B 69, 121401 (2004).

[16] N. Nilius, T. M. Wallis, and W. Ho, Science 297, 1853 (2002).

[17] M. Persson, Phys. Rev. B 70, 205420 (2004).

[18] S. Fölsch et al., Phys. Rev. Lett. 92, 056803 (2004).

[19] A. Bogicevic and D. R. Jennison, Phys. Rev. Lett. 82, 4050 (1999).

[20] V. V. Rivanenkov et al., Surf. Sci. 525, 173 (2003).

[21] M. Sterrer et al., J. Phys. Chem. B 110, 46 (2006). 